\title{
Capacidade reprodutiva e preferência da traça-das-crucíferas para diferentes brassicáceas
}

\author{
Sergio A De Bortoli ${ }^{1}$; Alessandra M Vacari ${ }^{1}$; Roberto M Goulart ${ }^{1}$; Rafael F Santos ${ }^{1}$; Haroldo XL Volpe ${ }^{1}$; \\ Antonio S Ferraudo ${ }^{2}$ \\ ${ }^{1}$ UNESP-FCAV, Dep ${ }^{\text {to }}$ Fitossanidade, Lab. Biologia e Criação de Insetos, 14884-900 Jaboticabal-SP, bortoli@fcav.unesp.br; ${ }^{2}$ UNESP- \\ FCAV, Dep ${ }^{\mathrm{to}}$ Ciências Exatas, amvacari@gmail.com; rm_goulart@yahoo.com; anaorfa@bol.com.br; hxlvolpe@ig.com.br; fsajago@gmail.com
}

\section{RESUMO}

O objetivo desta pesquisa foi comparar diferentes cultivares de brassicáceas em relação à capacidade reprodutiva e preferência para alimentação e oviposição da traça-das-crucíferas. Os experimentos foram realizados utilizando-se as cultivares de repolho Midori, Chato-de-Quintal, híbridos da Top Seed ${ }^{\circledR}$ - Agristar (TPC 308, TPC 681 e TPC 668), couve-flor Bola de Neve, couve brócolis Ramoso Piracicaba Precoce e couve Manteiga da Geórgia, sendo esta última utilizada como padrão de suscetibilidade. Por meio dos dados biológicos de $P$. xylostella foram estimados os parâmetros necessários para a construção de tabela de vida de fertilidade, para comparação das cultivares testadas em relação à capacidade reprodutiva da praga. Em gaiolas de criação da traça-das-crucíferas foram colocados quatro quartos de folha, dois a dois, que justapostos formavam um círculo de $8 \mathrm{~cm}$ de diâmetro, confrontando-se os materiais dois a dois. As partes foram dispostas equidistantemente, para realização do teste de dupla chance de escolha (preferência para alimentação e oviposição) e de múltipla chance de escolha, confrontando todos os substratos (preferência para alimentação). As cultivares que proporcionaram melhor desenvolvimento e reprodução para a traça-das-crucíferas foram couve Manteiga da Geórgia e couve brócolis. Para preferência alimentar constatou-se alta suscetibilidade em couve Manteiga e TPC 681 e para preferência de oviposição alta preferência para couve-flor Bola de Neve. Com isso, sugere-se a divisão das cultivares estudadas em quatro classes distintas: repolho Midori como moderadamente resistente (MR); couve-flor Bola de Neve e repolho Chato-de-Quintal como suscetíveis (S); couve brócolis, TPC308, TPC681 e TPC668 como moderadamente suscetíveis (MS); e couve Manteiga como altamente suscetível (AS).

Palavras-chave: Plutella xylostella, tabela de vida de fertilidade, biologia de insetos, preferência para alimentação, preferência para oviposição, entomologia agrícola.

\begin{abstract}
Reproductive capacity and preference of the diamondback moth feeding on different brassicacea
\end{abstract}

We compared different cultivars of crucifer in relation to reproductive capacity and preference for feeding and oviposition of the diamondback moth. The experiments were carried out with the following cultivars: Midori and Chato-de-Quintal cabbage, hybrids of cabbage Top Seed® - Agristar (TPC 308, TPC 681 and TPC 668), cauliflower Bola de Neve, broccolis Ramoso Piracicaba Precoce and collard green Manteiga da Geórgia, being this last one used as the susceptible control. Through the biological parameters of P. xylostella we elaborated a fertility life table, Comparing the cultivars in relation to the pest reproductive capacity. Four leaf parts were placed in diamondback moth rearing chambers, being two leaves of the treatment and two of the control which, put together formed a circle with $8 \mathrm{~cm}$ in diameter. The parts were disposed in an equidistantly form, to perform the dual-choice test (feeding and oviposition preference) and multiple-choice test, confronting all substrates (feeding preference). The cultivars that provided better development and reproduction for the diamondback moth were collard green Manteiga da Georgia and broccolis Ramoso Piracicaba Precoce. For feeding preference high susceptibility was verified in collard green Manteiga da Geórgia and TPC 681 and for oviposition preference high preference was observed for cauliflower Bola de Neve. Four different groups of cultivars could be formed: cabbage Midori as moderately resistant (MR); cauliflower Bola de Neve and cabbage Chato-de-Quintal as susceptible (S); broccolis, TPC 308, TPC 681 and TPC 668 as moderately susceptible (MS); and collard green as highly susceptible (HS).

Keywords: Plutella xylostella, fertility life table, biology of insects, feeding preference, oviposition preference, agricultural entomology.

(Recebido para publicação em 23 de junho de 2010; aceito em 25 de março de 2011) (Received on June 23, 2010; accepted on March 25, 2011)

\begin{abstract}
$\mathrm{A}$ traça-das-crucíferas, Plutella xylostella (L.) (Lepidoptera: Plutellidae), é o principal inseto praga de brassicáceas em áreas tropicais no mundo (Dickson et al., 1990), devido principalmente ao seu ciclo biológico curto e alto potencial reprodutivo, o que determina grande número anual de gerações nas regiões tropicais (França et al., 1985). As práticas culturais utilizadas,
\end{abstract}

como plantios sucessivos e não eliminação dos restos de culturas aumentam o potencial de dano da praga, devido à possibilidade de sua multiplicação contínua. Para reduzir os prejuízos, muitos produtores têm optado pelo controle químico, e muitas vezes um grande número de pulverizações é realizado para o controle do inseto. Essa prática tem se mostrado ineficaz. As aplicações inten- sivas de inseticidas selecionam populações resistentes e dificultam ainda mais seu manejo (Chen et al., 2008; Wang et al., 2010). Assim, alternativas têm sido buscadas para seu controle (Castelo Branco \& França, 2001).

Uma dessas alternativas seria o emprego de cultivares resistentes. Cultivares resistentes têm assumido papel relevante no manejo da traça-das-crucí- 
feras (Ulmer et al., 2002; Chagas Filho et al., 2010). Os testes de preferência são os mais frequentemente realizados e a resistência da cultura ao inseto está relacionada às características das folhas como coloração, cerosidade e brilho de diversos hospedeiros naturais (Harrison \& Brubaker, 1943; Justus \& Mitchell, 1996; Picoaga et al., 2003). Porém, pouco se conhece sobre a preferência alimentar e de oviposição dessa praga em diferentes cultivares de brassicáceas. O conhecimento das cultivares que apresentam resistência por antixenose pode auxiliar no manejo da praga.

Sendo assim, o estudo da biologia e testes de preferência em diferentes cultivares são importantes para que se possa determinar aquelas menos propícias para o desenvolvimento e reprodução da praga (Thuler et al., 2007; Badenes-Perez et al., 2010). Objetivou-se com esta pesquisa comparar diferentes cultivares de brassicáceas em relação à capacidade reprodutiva e à preferência para alimentação e oviposição da traça-das-crucíferas.

\section{MATERIAL E MÉTODOS}

Os experimentos foram conduzidos em sala climatizada a $25 \pm 1^{\circ} \mathrm{C}$, umidade relativa de $70 \pm 10 \%$ e fotofase de 12 h. As cultivares foram plantadas em área experimental com solo corrigido, segundo a necessidade de plantio. Foi realizada adubação de cobertura com a fórmula 4-14-8 (NPK) 20 dias após o transplante. As plantas foram constantemente irrigadas nos períodos de estiagem durante $1 \mathrm{~h}$ por dia. Foram utilizadas as cultivares: Brassica oleracea acephala - couve Manteiga da Geórgia, considerada padrão de suscetibilidade (Thuler et al., 2007); couve-flor Bola de Neve; couve brócolis Ramoso Piracicaba Precoce, utilizado somente para elaboração dos parâmetros da tabela de vida de fertilidade; $B$. oleracea capitata - repolho Chato-de-Quintal; e os híbridos - repolho Midori; repolho TPC668, TPC308 e TPC681. Ovos de P. xylostella foram retirados da criação estoque e os insetos foram mantidos nessas cultivares durante uma geração para condicionamento e, posteriormente, procedeu-se à experimentação.
Tabela de vida de fertilidade Para determinação das características biológicas de P. xylostella, para cada cultivar foram utilizadas 15 placas de Petri (9 cm de diâmetro), com um disco de folha de $8 \mathrm{~cm}$ de diâmetro, sobre papel filtro levemente umedecido com água destilada. Sobre os discos foliares foram colocadas 12 lagartas de primeiro estádio de $P$. xylostella, recém eclodidas e, posteriormente as placas foram fechadas com filme plástico, para manter a umidade e evitar a fuga dos insetos. Cada placa de Petri foi considerada uma repetição. As avaliações da viabilidade e duração larval iniciaram-se a partir do terceiro dia, em razão do hábito minador do inseto no primeiro estádio. As lagartas foram mantidas nas placas até atingirem a fase de pupa. Em segui$\mathrm{da}$, os insetos foram transferidos para placas de plástico com poços (idênticas àquelas utilizadas para testes ELISA $^{\circledR}$ ), sendo observados até a emergência dos adultos. Esse procedimento permitiu a obtenção da duração pupal, a viabilidade e a razão sexual dos insetos que emergiram. Adultos emergidos foram sexados e transferidos para gaiolas de postura, com quatro repetições por substrato, tendo-se colocado dois casais por gaiola. Nessa fase foi observada a fecundidade das fêmeas (número de ovos por fêmea), durante três dias (período de posturas viáveis) e a longevidade dos adultos. Utilizando-se os dados biológicos obtidos foram estimados os parâmetros necessários para a construção de tabela de vida de fertilidade, segundo Birch (1948), Silveira Neto et al. (1976), Southwood (1978) e Price (1984), que são: $x$ = ponto médio de cada idade das fêmeas parentais, idade esta considerada desde a fase de ovo; $1 \mathrm{x}=$ expectativa de vida até a idade $\mathrm{x}$, expressa como uma fração de uma fêmea; $m x$ = fertilidade específica ou número de descendentes por fêmea produzidos na idade $\mathrm{x}$ e que originarão fêmeas; lx.mx = número total de fêmeas nascidas na idade x. Os parâmetros de crescimento resultantes da tabela de vida foram calculados de acordo com aqueles autores, sendo $\mathrm{R}_{\mathrm{o}}=$ taxa líquida de aumento populacional; $\mathrm{T}=$ tempo médio de geração; $\mathrm{r}_{\mathrm{m}}=$ taxa intrínseca de aumento; $\lambda=$ razão finita de aumento. Além desses parâmetros, foi também determinado o TD = tempo necessário para a população duplicar em número, segundo Krebs (1994).

Os dados foram submetidos à análise de variância, com comparação das médias pelo teste de Tukey $(\mathrm{p}<0,05)$.

Preferência para alimentação - Estes testes foram realizados em placas de Petri plásticas, com $15 \mathrm{~cm}$ de diâmetro. $\mathrm{O}$ fundo das placas foi coberto por um círculo de papel filtro levemente umedecido com água destilada para evitar o ressecamento das folhas.

Para o teste de dupla chance de escolha, no interior das placas foram dispostos equidistantemente dois discos de $2 \mathrm{~cm}$ de diâmetro de cada cultivar, confrontando-se duas cultivares por vez. No centro de cada placa foram colocadas dez lagartas de primeiro ínstar de $P$. xylostella. Para cada tratamento foram montadas cinco repetições. As avaliações foram feitas com 24 h. Para isso, foram contadas as lagartas presentes em cada um dos discos.

Para o teste de múltipla escolha, dentro de cada placa foram colocadas equidistantemente seis discos de folhas de brassicáceas, sendo um de cada cultivar, e no centro 20 lagartas de primeiro ínstar. $\mathrm{O}$ experimento foi composto por dez repetições. As avaliações foram feitas com $24 \mathrm{~h}$. Para isso, foram contadas as lagartas presentes em cada um dos discos.

Para o teste de dupla chance foi calculado o Índice de Preferência IP, de acordo com Kogan (1972), confrontando-se os hospedeiros dois a dois pela fórmula $\mathrm{IP}=2 \mathrm{~A} /(\mathrm{A}+\mathrm{T})$, onde $\mathrm{A}=$ número de lagartas de $P$. xylostella presentes na brassicácea da primeira cultivar confrontada e $\mathrm{T}=$ da segunda cultivar, nas comparações. Aos IP calculados foi adicionado/subtraído o valor do Erro Padrão da média (EP). Valores de $\mathrm{IP} \pm \mathrm{EP}>1$ indicam preferência pelo hospedeiro "A", enquanto valores de $\mathrm{IP} \pm \mathrm{EP}<1$, indicam preferência pelo hospedeiro " $\mathrm{T}$ ", sendo que valores onde $\mathrm{IP} \pm \mathrm{EP}=1$, indicam neutralidade, não existindo distinção pelos hospedeiros.

Para o teste de múltipla chance foi aplicada a análise de componentes principais (ACP) (Jackson, 1991) com uso do programa Statistica versão 7.0 (Statsoft Inc., 2004). A análise de componentes principais foi utilizada como 
análise exploratória, o que não necessita investigar as pressuposições de independência e normalidade.

Preferência para oviposição - Esta determinação foi realizada em gaiolas de criação da traça-das-crucíferas (Thuler, 2006). Foram colocados quatro quartos de um círculo de $8 \mathrm{~cm}$ de diâmetro das cultivares nas gaiolas, sendo dois quartos de uma cultivar e dois quartos de outra, sempre confrontando-se os genótipos dois a dois (teste de dupla chance de escolha). Dentro de cada gaiola foram colocados dois casais de adultos de $P$. xylostella e para cada tratamento foram montadas quatro repetições. Os ovos em cada parte da folha foram contados após $24 \mathrm{~h}$ de exposição aos adultos, para determinação do índice de preferência. Para a análise dos dados foi calculado o Índice de Preferência IP, de acordo com Kogan (1972), conforme descrito no subitem anterior.

\section{RESULTADOS E DISCUSSÃO}

A determinação do aumento populacional devido à capacidade reprodutiva é um componente crucial no estudo de populações, sendo que esse aumento pode ser descrito pela tabela de vida apresentando o potencial reprodutivo das fêmeas em diferentes tempos (Medeiros et al., 2000). Os parâmetros populacionais da tabela de vida de $P$. xylostella foram afetados pelas diferentes brassicáceas (Tabela 1). A taxa líquida de reprodução $\left(\mathrm{R}_{\mathrm{o}}\right.$ ) foi maior para $\mathrm{o}$ tratamento couve Manteiga. Já para o tempo médio de geração $(\mathrm{T})$ ocorreu o oposto, sendo que o maior valor foi encontrado em repolho Midori. A taxa intrínseca de crescimento populacional $\left(\mathrm{r}_{\mathrm{m}}\right)$ e a razão finita de crescimento populacional $(\lambda)$ foram superiores para couve Manteiga. O tempo necessário para a população duplicar em número (TD) foi menor em couve Manteiga. A tabela de vida de fertilidade de P. xylostella mostrou que couve Manteiga, couve brócolis, TPC308, TPC681 e TPC668 proporcionaram maior taxa líquida de reprodução, o que indica maior produção de descendentes por geração. A taxa intrínseca de crescimento populacional é representada pela combinação de $\mathrm{R}_{0} \mathrm{e} \mathrm{T}$, e quando a praga retarda a produção de
Tabela 1. Parâmetros relativos às tabelas de vida de fertilidade de Plutella xylostella, em diferentes cultivares de brassicáceas (fertility life table parameters of Plutella xylostella, in different crucifer cultivars). Jaboticabal, UNESP, 2010.

\begin{tabular}{lccccc}
\hline Tratamentos & $\mathbf{R}_{0}$ & $\mathbf{T}$ & $\mathbf{r}_{\mathrm{m}}$ & \multicolumn{1}{c}{$\lambda$} & $\mathbf{T D}$ \\
\hline Couve manteiga & $60,8 \mathrm{a}^{1}$ & $17,9 \mathrm{~d}$ & $0,2274 \mathrm{a}$ & $1,2554 \mathrm{a}$ & $3,1 \mathrm{~d}$ \\
TPC668 & $20,1 \mathrm{bc}$ & $18,8 \mathrm{c}$ & $0,1593 \mathrm{bc}$ & $1,1727 \mathrm{bc}$ & $4,3 \mathrm{c}$ \\
TPC681 & $23,2 \mathrm{bc}$ & $18,7 \mathrm{c}$ & $0,1676 \mathrm{~b}$ & $1,1825 \mathrm{~b}$ & $4,1 \mathrm{c}$ \\
RCQ & $8,8 \mathrm{c}$ & $17,9 \mathrm{~d}$ & $0,1216 \mathrm{~d}$ & $1,1293 \mathrm{~d}$ & $5,7 \mathrm{~b}$ \\
RM & $3,8 \mathrm{c}$ & $20,9 \mathrm{a}$ & $0,0643 \mathrm{~d}$ & $1,0664 \mathrm{e}$ & $10,8 \mathrm{a}$ \\
CFBN & $12,6 \mathrm{c}$ & $18,9 \mathrm{c}$ & $0,1329 \mathrm{c}$ & $1,1422 \mathrm{~cd}$ & $5,2 \mathrm{~b}$ \\
TPC308 & $28,7 \mathrm{bc}$ & $18,9 \mathrm{c}$ & $0,1753 \mathrm{~b}$ & $1,1917 \mathrm{~b}$ & $4,0 \mathrm{c}$ \\
Brócolis & $38,5 \mathrm{~b}$ & $19,7 \mathrm{~b}$ & $0,1847 \mathrm{~b}$ & $1,2030 \mathrm{~b}$ & $3,8 \mathrm{c}$ \\
\hline F & $14,34^{* *}$ & $48,10^{* *}$ & $46,04^{* *}$ & $43,97^{* *}$ & $11,45^{* *}$ \\
CV (\%) & 33,68 & 1,10 & 6,78 & 1,13 & 5,99 \\
\hline
\end{tabular}

${ }^{1}$ Médias \pm intervalo de confiança, seguidas pela mesma letra na mesma coluna, não diferem entre si pelo teste de Tukey, ao nível de $5 \%$ de probabilidade; $\mathrm{R}_{\mathrm{o}}=$ taxa líquida de reprodução (fêmea/fêmea); $\mathrm{T}=$ tempo médio de geração (dias); $\mathrm{r}_{\mathrm{m}}=$ taxa intrínseca de crescimento populacional; $\lambda=$ taxa finita de crescimento populacional (fêmeas/dia); $\mathrm{TD}=$ tempo necessário para a população duplicar em número (means \pm confiance interval, followed by the same letter in the same column, do not differ from each other for the Tukey test, at the level of $5 \%$ of probability; $\mathrm{R} 0=$ net reproductive rate; $\mathrm{T}=$ mean generation time; $\mathrm{rm}=$ intrinsic rate of population increase; $\lambda=$ finite rate of increase; DT = population doubling time).

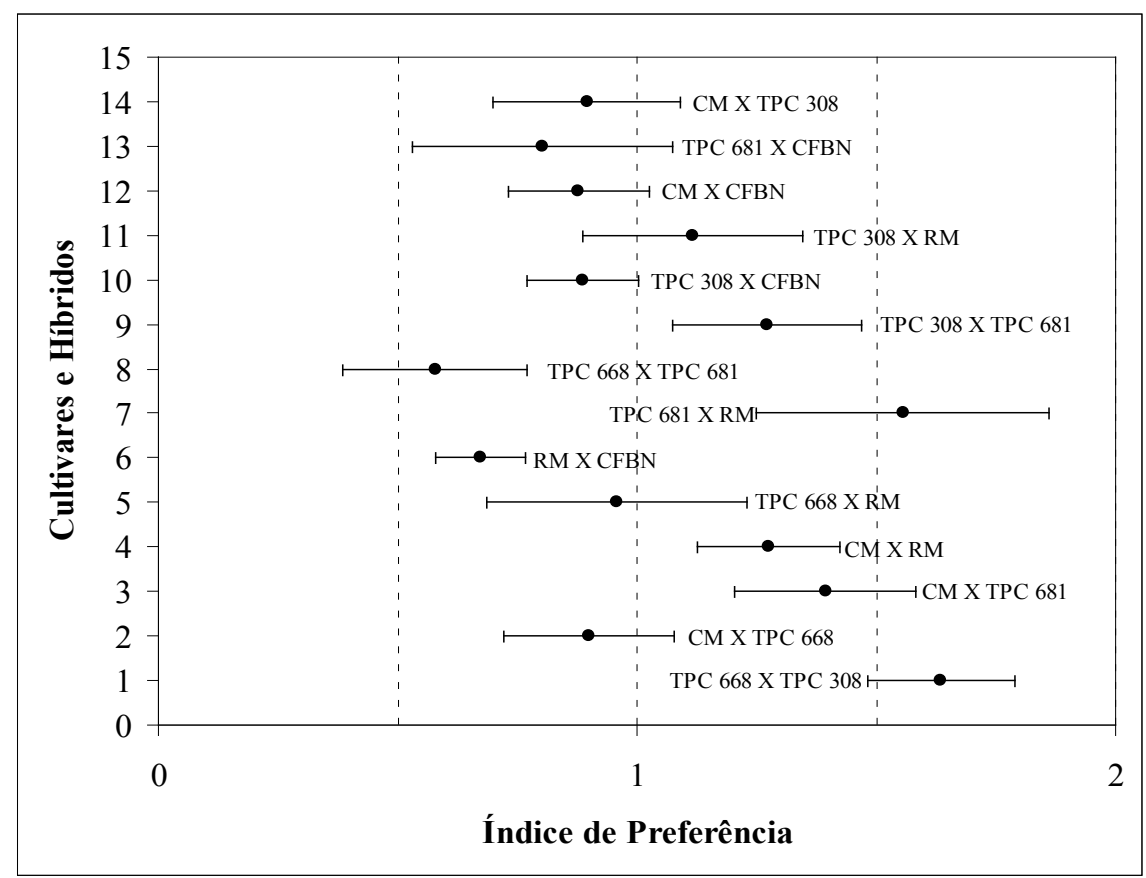

Figura 1. Índice de preferência (IP) de alimentação de Plutella xylostella, comparando as cultivares duas a duas. Valores de $\mathrm{IP} \pm \mathrm{EP}>1$ indicam preferência pelo hospedeiro "A", enquanto valores de $\mathrm{IP} \pm \mathrm{EP}<1$, indicam preferência pelo hospedeiro "T", sendo que valores onde $\mathrm{IP} \pm \mathrm{EP}=1$, indicam neutralidade. $\mathrm{RM}=$ repolho Midori; híbridos de repolho TPC 308, TPC 681, TPC 668; CFBN= couve flor Bola de Neve; RCQ= repolho Chato de Quintal; $\mathrm{CM}=$ couve Manteiga da Geórgia (feeding preference index of Plutella xylostella, comparing the cultivars by pairs. $\mathrm{IP} \pm \mathrm{SE}$ values $>1$ indicate preference for host " $\mathrm{A}$ ", $\mathrm{IP} \pm \mathrm{SE}$ values $<1$ indicate preference for host " $T$ ", and where $\mathrm{IP} \pm \mathrm{SE}$ values $=1$ indicate neutrality. $\mathrm{RM}=$ cabbage Midori; cabbage hybrids TPC 308, TPC 681, TPC 668; CFBN= cauliflower Bola de Neve; $\mathrm{RCQ}=$ cabbage Chato de Quintal; $\mathrm{CM}=$ collard Manteiga da Geórgia). Jaboticabal, UNESP, 2010. 


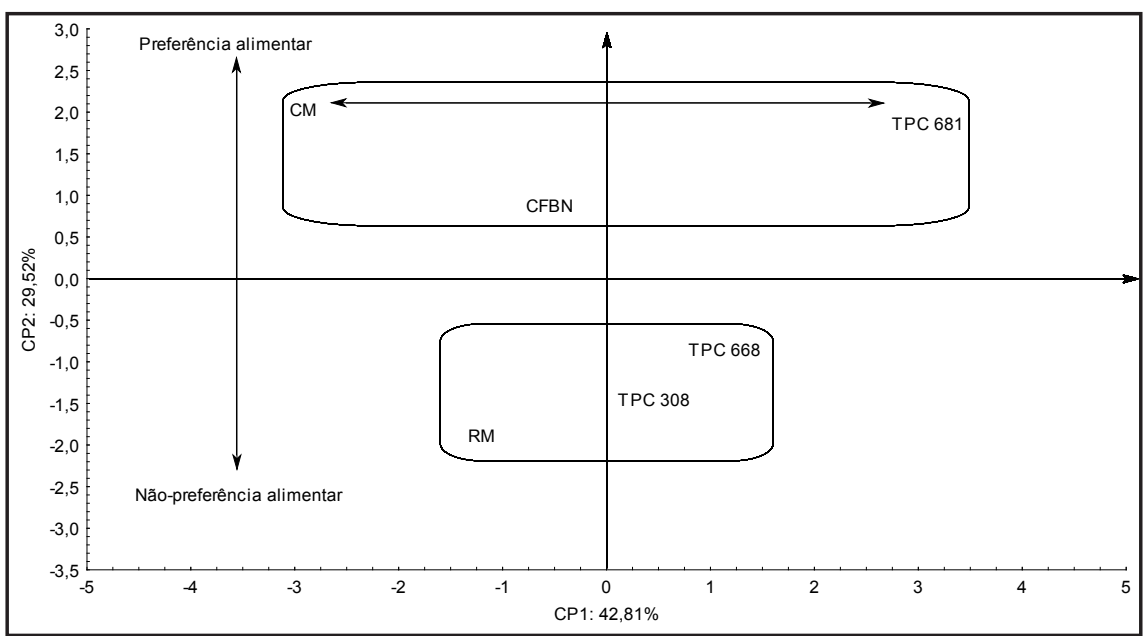

Figura 2. Ordenação em grupos das brassicáceas em teste de múltipla chance de escolha para alimentação, nos dois primeiros componentes principais. $\mathrm{RM}=$ repolho Midori; híbridos de repolho TPC 308, TPC 681, TPC 668; $\mathrm{CFBN}=$ couve flor Bola de Neve; $\mathrm{RCQ}=$ repolho Chato de Quintal; $\mathrm{CM}=$ couve Manteiga da Geórgia (group of crucifers formed in multiple choice feeding test in the first two main components. $\mathrm{RM}=$ cabbage Midori; cabbage hybrids TPC 308, TPC 681, TPC 668; CFBN= cauliflower Bola de Neve; RCQ= cabbage Chato de Quintal; CM= collard Manteiga da Geórgia). Jaboticabal, UNESP, 2010.

descendentes no mesmo espaço de tempo e/ou apresenta maior longevidade, os valores de $r_{m}$ tornam-se menores. Este fato foi observado para fêmeas criadas em repolho Chato de Quintal e repolho Midori, que também apresentaram menores taxas líquidas de reprodução $\left(\mathrm{R}_{0}\right)$, por apresentar maior longevidade e produção de descendentes distribuídos ao longo da vida. As cultivares comerciais de brassicáceas que proporcionaram melhor desenvolvimento e reprodução para a traça-das-crucíferas foram couve Manteiga e couve brócolis. Thuler et al. (2007) também observaram que diferentes cultivares de brassicáceas influenciaram características biológicas de P. xylostella utilizadas para elaboração dos parâmetros da tabela de vida de fertilidade, como duração e viabilidade, sendo que, os autores verificaram que o repolho Chato-de-Quintal proporcionou maior período larval (8,2 dias) e pupal (4,2 dias), em relação à couve Manteiga da Geórgia (6,8 e 3,3 dias). Além disso, os autores também verificaram que larvas e pupas criadas em repolho tiveram menor viabilidade $(61,3$ e $70,7 \%)$ do que em couve (89,3 e $86,1 \%)$.

Quanto a preferência para alimentação, couve Manteiga foi preferida por P. xylostella quando comparada com repolho Midori e o híbrido TPC 681, ocorrendo condição de neutralidade quando esta cultivar foi comparada à TPC 308, couve-flor Bola de Neve e TPC 668 (Figura 1). O híbrido de repolho TPC 308 foi preferido para alimentação das lagartas quando comparado ao TPC 681, porém houve neutralidade em relação ao repolho Midori e à couve-flor Bola de Neve. Ocorreu condição de neutralidade com TPC 681, em relação ao repolho Midori. A neutralidade se repetiu quando TPC 668 foi confrontado com TPC 308 e na comparação de TPC 668 e repolho Midori. Finalmente pode-se dizer que TPC 681 foi preferida em relação à TPC 668, não havendo preferência quando comparada à couve-flor Bola de Neve (Figura 1). Com base nos IP's, verifica-se alta suscetibilidade para couve Manteiga e TPC 681, suscetibilidade moderada para couve-flor Bola de Neve e resistência moderada para TPC 668, 308 e repolho Midori. Para o teste de múltipla chance de escolha nota-se similaridade entre os híbridos de repolho TPC 668 e TPC 308, sendo eles igualmente procurados por P. xylostella. Porém, neles foi encontrado baixo número de larvas nos discos, mostrando resistência do tipo não-preferência para alimentação. Tal fato é confirmado pela condição de neutralidade entre esses híbridos no IP (Figura 1). Isso corrobora a proximidade deles na análise de componentes principais, situando-se juntamente com repolho Midori, no grupo das brassicáceas mais resistentes (Figura 2). Por outro lado, verifica-se dissimilaridade entre o híbrido TPC 681 e a testemunha, representada pela couve Manteiga (padrão de suscetibilidade) (Figura 2). Isso ocorre porque quando uma dessas brassicáceas é altamente procurada para alimentação por $P$. xylostella, a procura pela outra se torna baixa, e vice-versa. Porém, ambas as cultivares são suscetíveis ao ataque da lagarta e em menor escala couve-flor Bola de Neve (Figura 2). Isso mostra a preferência para alimentação de $P$. xylostella a esses substratos, situação esta caracterizada pela localização na parte superior do gráfico, porém, em lados opostos, devido à dissimilaridade entre eles. Assim, pode-se verificar alta suscetibilidade nos substratos couve Manteiga e TPC 681, suscetibilidade moderada na couve-flor Bola de Neve, suscetibilidade em TPC 668 e 308 e resistência moderada para repolho Midori.

Em relação à preferência para oviposição, o híbrido de repolho TPC 308 foi preferido por P. xylostella quando comparado com TPC 668 e com o híbrido TPC 681, ocorrendo condição de neutralidade em relação à couve Manteiga e repolho Midori (Figura 3). Couve-flor Bola de Neve foi preferida para oviposição quando comparada a TPC 308, couve Manteiga, TPC 668, porém houve neutralidade quando confrontada com repolho Midori e TPC 681. A neutralidade se repetiu quando couve Manteiga foi relacionada com repolho Midori e TPC 668 (Figura 3). TPC 681 foi preferida em relação à couve Manteiga e TPC 668, enquanto repolho Midori foi preferido pelos adultos para oviposição em relação à TPC 681 e TPC 668 (Figura 3). Com base nesse índice verifica-se alta preferência da praga a cultivar de couve-flor Bola de Neve, preferência moderada aos materiais TPC 681, TPC 308, repolho Midori e couve Manteiga e baixa preferência para oviposição no híbrido TPC 668.

Com base nos resultados verifica-se que, das cultivares testadas, couve Manteiga é altamente suscetível. A cultivar couve-flor Bola de Neve, apesar de apresentar alta preferência para oviposição foi suscetível em relação aos parâmetros 


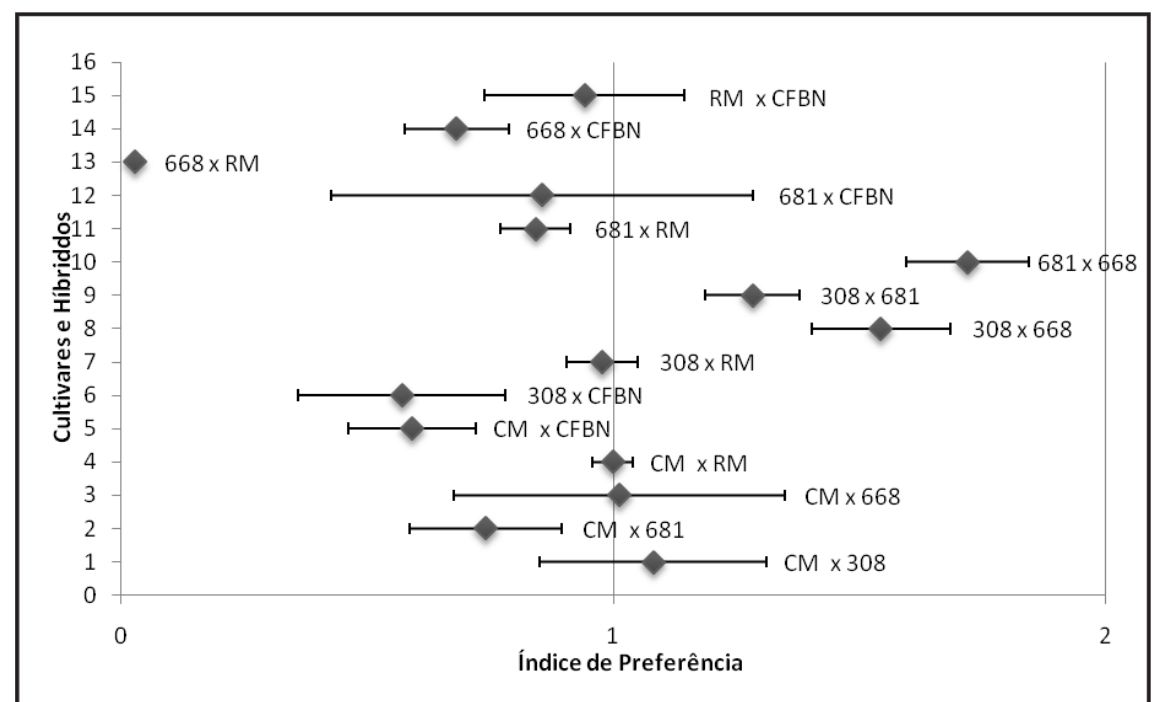

Figura 3. Índice de preferência (IP) de oviposição de Plutella xylostella, comparando as cultivares duas a duas. Valores de $\mathrm{IP} \pm \mathrm{EP}>1$ indicam preferência pelo hospedeiro "A", enquanto valores de $\mathrm{IP} \pm \mathrm{EP}<1$, indicam preferência pelo hospedeiro " $\mathrm{T}$ ", sendo que valores onde $\mathrm{IP} \pm \mathrm{EP}=1$, indicam neutralidade. $\mathrm{RM}=$ repolho Midori; híbridos de repolho TPC 308, TPC 681, TPC 668; CFBN= couve flor Bola de Neve; $\mathrm{RCQ}=$ repolho Chato de Quintal; $\mathrm{CM}=$ couve Manteiga da Geórgia (oviposition preference index of Plutella xylostella, comparing the cultivars by pairs. $\mathrm{IP} \pm \mathrm{SE}$ values $>1$ indicate preference for host " $\mathrm{A}$ ", $\mathrm{IP} \pm \mathrm{SE}$ values $<1$ indicate preference for host " $\mathrm{T}$ ", and where $\mathrm{IP} \pm \mathrm{SE}$ values $=1$ indicate neutrality. $\mathrm{RM}=$ cabbage Midori; cabbage hybrids TPC 308, TPC 681, TPC 668; CFBN= cauliflower Bola de Neve; $\mathrm{RCQ}=$ cabbage Chato de Quintal; $\mathrm{CM}=$ collard Manteiga da Geórgia). Jaboticabal, UNESP, 2010

da tabela de vida de fertilidade e neutra quando a comparação se deu com couve Manteiga que apresentou suscetibilidade moderada. O híbrido TPC 668, considerado como moderadamente suscetível em relação aos parâmetros da tabela de vida de fertilidade e em relação à preferência de alimentação, proporcionou menor preferência para oviposição quando comparado aos demais materiais. Portanto, a utilização desse híbrido em plantios com alta ocorrência da praga pode resultar em menor número de ovos nas plantas, minimizando o aumento da população no decorrer das gerações. Dentre as cultivares capitata, o repolho Midori é o mais indicado para plantio, por ser moderadamente resistente à traça-das-crucíferas, e possivelmente pode ser mais rentável ao agricultor por proporcionar menores gastos com o manejo dessa praga, já que a aplicação de inseticidas encarece o produto. É ainda possível amenizar o ataque da praga com a utilização da rotação de culturas com outras famílias de plantas a fim de reduzir a população da praga no campo. Outra alternativa para amenizar o ataque da praga seria intercalar uma cultivar resistente com uma suscetível, com o objetivo de diminuir o potencial biótico da praga em campo. Tagliari (2007) verificou em teste com chance de escolha que couve Manteiga de Ribeirão Pires e couve Manteiga de São José se destacaram entre outros genótipos de couve como os mais atrativos para consumo e oviposição de $P$. xylostella.

Das cultivares testadas, o repolho Midori é a menos propícia para o desenvolvimento e reprodução da traça-das-crucíferas, além de ser a menos preferida pela praga. Com base nesses resultados, sugere-se a divisão das cultivares estudadas em quatro grupos distintos, na seguinte classificação: repolho Midori como moderadamente resistente (MR); couve brócolis, TPC308, TPC681 e TPC668 como moderadamente suscetíveis (MS); couve-flor Bola de Neve e repolho Chato-de-Quintal como suscetíveis ( $\mathrm{S}$ ); e couve Manteiga como altamente suscetível (AS).

Em conclusão, a presente pesquisa mostrou que $P$. xylostella pode desenvolver e reproduzir-se em todas as cultivares testadas, porém a planta hospedeira afeta significativamente as características biológicas e a preferência da traça-das-crucíferas. Dentre as brassicáceas testadas, as cultivares capitata (repolho) são as mais indicadas para plantio por apresentarem mais características de resistência do que as cultivares acephala (couve). Finalmente, o presente estudo forneceu informações fundamentais para o desenvolvimento de estratégias que são instrumentos para o Manejo Integrado da traça-das-crucíferas em brassicáceas. Embora os insetos sejam expostos a condições adversas na natureza, estudos em condições controladas em laboratório podem oferecer sinais valiosos sobre a dinâmica populacional de uma determinada espécie. É necessário investigar o impacto de fatores ecológicos, como a planta hospedeira, no desempenho de $P$. xylostella em condições de campo para saber o efeito sobre a sua preferência e história de vida.

\section{REFERÊNCIAS}

BADENES-PEREZ FR; REICHELT M; HECKEL DG. 2010. Can sulfur fertilization improve the effectiveness of trap crops for diamondback moth, Plutella xylostella (L.) (Lepidoptera: Plutellidae)? Pest Management Science 66: 832-838.

BIRCH LC. 1948. The intrinsic rate of natural increase of an insect population. Journal Animal Ecology 17: 15-26.

CASTELO BRANCO M; FRANÇA FH. 2001. Traça-das-crucíferas, Plutella xylostella (Lepidoptera: Yponomeutidae). In: VILELA EF; ZUCCHI RA; CANTOR F. (eds). Pragas introduzidas no Brasil. Ribeirão Preto: Holos. p. 85-89.

CHAGAS FILHO NR; BOIÇA JÚNIOR AL; ALONSO TF. 2010. Biologia de Plutella xylostella L. (Lepidoptera: Plutellidae) em cultivares de couve-flor. Neotropical Entomology 39: 253-259.

CHEN RX; ZHANG F; HUANGFU WG; YAO HY; ZHOU JB; KUHLMANN U. Reproductive attributes of the eulophid Oomyzus sokolowskii, a biological control agent of diamondback moth, Plutella xylostella (Lepidoptera: Plutellidae). Biocontrol Science and Technology 18: 753-765.

DICKSON MH; SHELTON AM; EIGENBRODE SD; VAMOSY ML; MORAM. 1990. Selection for resistance to diamondback moth (Plutella xylostella) in cabbage. Hortscience 25: 1643 1646.

FRANÇA FH; CORDEIRO CMT; GIORDANO LB; RESENDE AM. 1985. Controle da traça-das-crucíferas em repolho. Horticultura Brasileira 3: 47-53.

HARRISON PK; BRUBAKER RW. 1943. The relative abundance of cabbage caterpillars 
on cole crops grown under similar condition. Journal of Economic Entomology 36: 589-592.

JACKSON JE. 1991. A user's guide to principal components. New York: Wiley, 569p.

JUSTUS KA; MITCHELL BK. 1996. Oviposition site selection by the diamondback moth, Plutella xylostella (L.) (Lepidoptera: Plutellidae). Journal of Insect Behavior 9: 887-897.

KOGAN M. 1972. Feeding and nutrition of insects associated with soybeans. 2. Soybean resistance and host preferences of Mexican bean beetle, Epilachna varivestis. Annals of Entomological Society of America 65: 675-683.

KREBS CJ. 1994. Ecology: The experimental analysis of distribution and abundance. New York: Harper \& Row, 801p.

MEDEIROS RS; RAMALHO FS; LEMOS WP; ZANUNCIO JC. 2000. Age-dependent fecundity and life-fertility tables for Podisus nigrispinus (Dallas) (Het., Pentatomidae).
Journal of Applied Entomology 124: 319-324.

PICOAGA A; CARTEA ME; SOENGAS P; MONETTI L; ORDÁS A. 2003. Resistance of kale populations to lepidopterous pests in northwestern Spain. Journal of Economic Entomology 96: 143-147.

PRICE PW. 1984. Insect ecology. 2ª ed. New York: John Willey, 607p.

SILVEIRA NETO S; NAKANO O; BALDIN D; VILLANOVA NA. 1976. Manual de ecologia dos insetos. São Paulo: Agronômica Ceres, 419p.

SOUTHWOOD TRE. 1978. Ecological methods. $2^{\underline{a}}$ ed. London: Chapman and Hall, 524p.

STATSOFT INC. 2004. Statistica: data analysis software system, version 7. Tulsa. Disponível em http://www.statsoft.com/

TAGLIARI SRA. 2007. Não-preferência para oviposição, alimentação e antibiose de Plutella xylostella (L., 1758) (Lepidoptera: Plutellidae) por genótipos de couve (Brassica oleracea L. var. acephala D.C.). Jaboticabal: Unesp - FCAV. 76p (Dissertação mestrado).

THULER RT. 2006. Plutella xylostella (L.) (Lepidoptera: Plutellidae): táticas para o manejo integrado em brássicas. Jaboticabal : UNESP-FCAV. 80p (Tese doutorado).

THULER RT; DE BORTOLI SA; HOFFMANNCAMPO CB. 2007. Classificação de cultivares de brássicas com relação à resistência à traçadas-crucíferas e à presença de glucosinolatos. Pesquisa Agropecuária Brasileira 42: 467474.

ULMER BC; GILLOTT C; WOODS D; ERLANDSON M. 2002. Diamond moth, Plutella xylostella $(\mathrm{L}$.$) , feeding and oviposition$ preferences on glossy and waxy Brassica rapa (L.) lines. Crop protection 21: 327-331.

WANG X; LI X; SHEN A; WU Y. Baseline susceptibility of the diamondback moth (Lepidoptera: Plutellidae) to chlorantraniliprole in China. Journal of Economic Entomology 103: 843-848. 